\title{
Dasatinib response in acute myeloid leukemia is correlated with FLT3/ITD, PTPN11 mutations and a unique gene expression signature
}

Sigal Tavor ${ }^{1}$ Tali Shalit ${ }^{2}$ Noa Chapal Ilani ${ }^{3}$ Yoni Moskovitz ${ }^{3}$ Nir Livnat, ${ }^{3}$ Yoram Groner, ${ }^{4}$ Haim Barr, ${ }^{2}$ Mark D. Minden, ${ }^{5,6,7,8}$ Alexander Plotnikov, ${ }^{2}$ Michael W. Deininger, ${ }^{9,10}$ Nathali Kaushansky ${ }^{3, \#}$ and Liran I. Shlush ${ }^{3,5,11, \#}$

${ }^{1}$ Hemato-Oncology Department, Assuta Medical Center, Tel Aviv, Israel; ${ }^{2} \mathrm{G}-$ INCPM, Weizmann Institute of Science, Rehovot, Israel; ${ }^{3}$ Department of Immunology, Weizmann Institute of Science, Rehovot, Israel; ${ }^{4}$ Department of Molecular Genetics, Weizmann Institute of Science, Rehovot, Israel; ${ }^{5}$ Princess Margaret Cancer Centre, University Health Network Toronto, Ontario, Canada; ${ }^{6}$ Department of Medical Biophysics, University of Toronto, Toronto, Ontario, Canada; 'Department of Medicine, University of Toronto, Toronto, Ontario, Canada; ${ }^{8}$ Division of Medical Oncology and Hematology, University Health Network, Toronto, Ontario, Canada; ${ }^{9}$ Division of Hematology and Hematologic Malignancies, University of Utah, Salt Lake City, UT, USA; ${ }^{10} \mathrm{Huntsman}$ Cancer Institute, University of Utah, Salt Lake City, UT, USA and ${ }^{11}$ Division of Hematology, Rambam Healthcare Campus, Haifa, Israel

"NK and LIS contributed equally as co-senior authors.

\section{ABSTRACT}

$\mathrm{N}$ ovel targeted therapies improve the survival of specific subgroups (defined by genetic variants) of patients with acute myeloid leukemia (AML), validating the paradigm of molecularly targeted therapy. However, identifying correlations between AML molecular attributes and effective therapies is challenging. Recent advances in highthroughput, in vitro drug sensitivity screening applied to primary AML blasts were used to uncover such correlations; however, these methods cannot predict the response of leukemic stem cells. Our study aimed to predict in vitro response to targeted therapies, based on molecular markers, with subsequent validation in leukemic stem cells. We performed ex vivo screening of sensitivity to 46 drugs on 29 primary AML samples at diagnosis or relapse. Using unsupervised hierarchical clustering analysis we identified a group with sensitivity to several tyrosine kinase inhibitors, including the multi-tyrosine kinase inhibitor, dasatinib, and searched for correlations between the response to dasatinib, exome sequencing and gene expression in our dataset and in the Beat AML dataset. Unsupervised hierarchical clustering analysis of gene expression resulted in clustering of dasatinib responders and non-responders. In vitro response to dasatinib could be predicted based on gene expression (area under the curve $=0.78$ ). Furthermore, mutations in FLT3/ITD and PTPN11 were enriched in the dasatinib-sensitive samples as opposed to mutations in TP53 which were enriched in resistant samples. Based on these results, we selected FLT3/ITD AML samples and injected them into NSG-SGM3 mice. Our results demonstrate that in a subgroup of FLT3/ITD AML (4 out of 9) dasatinib significantly inhibited leukemic stem cell engraftment. In summary we show that dasatinib has an anti-leukemic effect both on bulk blasts and, more importantly, on leukemic stem cells from a subset of AML patients that can be identified based on mutational and expression profiles. Our data provide a rational basis for clinical trials of dasatinib in a molecularly selected subset of AML patients.
Haematologica 2020

Volume 105(12):2795-2804

\section{Correspondence:}

LIRAN I. SHLUSH

liran.shlush@weizmann.ac.il

Received: October 16, 2019

Accepted: April 30, 2020.

Pre-published: May 21, 2020.

doi:10.3324/haematol.2019.240705

(C)2020 Ferrata Storti Foundation

Material published in Haematologica is covered by copyright. All rights are reserved to the Ferrata Storti Foundation. Use of published material is allowed under the following terms and conditions:

https://creativecommons.org/licenses/by-nc/4.0/legalcode. Copies of published material are allowed for personal or internal use. Sharing published material for non-commercial purposes is subject to the following conditions: https://creativecommons. org//icenses/by-nc/4.0/legalcode, sect. 3. Reproducing and sharing published material for commercial purposes is not allowed without permission in writing from the publisher. 


\section{Introduction}

AML is an aggressive myeloid neoplasm with complex and heterogeneous genetics that influence prognosis and treatment response. Furthermore, AML is a multi-stage disease with preleukemic, leukemic and late stages. ${ }^{1}$ Identification of AML-inducing mutations is required for accurate diagnosis and to tailor therapy according to the genetic profile of individual patients. Recent advances in AML targeted therapy based on driver mutations have improved overall survival.,3 Increased understanding of AML molecular pathophysiology provided new opportunities to target specific mutants, such as FLT3/ITD (FMSlike tyrosine kinase-3, internal tandem duplications) and IDH1/2. ${ }^{4 \cdot 6}$ Moreover, the BCL2 inhibitor, venetoclax, has increased activity in patients with mutated NPM1c and RAD 21, and in combination with hypomethylating agents targets leukemia stem cells (LSC). ${ }^{8}$ While these novel therapies can achieve prolonged remissions, most patients eventually relapse. Our recent studies suggest that the origins of AML relapse are heterogeneous, but relapsing clones invariably exhibit stem cell properties. ${ }^{9}$ Relapse usually originates from leukemic subclones that were present before therapy, and were either selected due to resistance mechanisms ${ }^{9,10}$ or gained competitiveness through further evolution, possibly due to exposure to chemotherapy. ${ }^{11}$ Targeted therapies can fail to prevent relapse by addressing one leukemic cell clone, while allowing other clones to expand and cause relapse. ${ }^{12}$ In order to prevent relapse, it is crucial to eradicate LSC in the early stages of treatment (induction/consolidation) before they expand and acquire additional mechanisms of resistance. It has been suggested that combination therapies that simultaneously target multiple leukemic subclones may overcome AML heterogeneity. However, combination therapy approaches are limited by tolerability, particularly in older individuals, and it is important to maximize efficacy and minimize toxicity by selecting the right drug for the right set of patients. Given the multitude of therapeutic options, it is essential to design clinical trials based on molecular markers that select for those patients likely to benefit.

Our latest studies demonstrated that, in certain AML patients in whom relapse originated from primitive cells, ${ }^{9}$ the cells from the time of diagnosis capable of leukemic engraftment in immunodeficient mice were identical to those that caused relapse. In these patients the bulk of cells were responsive to chemotherapy, but rare LSC that expanded in xenografts could drive relapse. Accordingly, drugs that inhibit the engrafting clones should be useful in preventing relapse, but screening a large number of drugs in a large number of samples in xenografts is not feasible. An alternative approach is to screen for drugs with known mechanistic effects against large and heterogeneous AML cohorts in vitro, which allows for high throughput screening of many drugs to establish correlations between response and molecular attributes of AML. Disadvantages include the lack of a microenvironment and immune system and the short duration of exposure to the drug, which reflects the inability of AML cells to grow in vitro. Short-term in vitro drug sensitivity assays have become feasible in recent years by mirroring normal hematopoietic stem and progenitor cell culture conditions. So far in vitro drug testing of primary AML samples have focused on predicting clinical outcome,,$^{13,14}$ and, in some cases, guiding individ- ualized therapy. ${ }^{15}$ Such personalized therapy is particularly important for patients with relapsed/refractory disease. ${ }^{16}$ In vitro drug studies identified biomarkers of response to specific drugs ${ }^{17}$ and clusters of AML samples with similar response profiles. ${ }^{18}$ In the current study, we studied in vitro drug sensitivity of primary AML samples from patients who achieved complete remission and compared our results to those of the Beat AML study. Our global aim was to identify therapies and biomarkers that could predict which drugs might be added to induction therapy to prevent relapse in specific subtypes of AML and then validate the results in vivo. We discovered that samples with in vitro sensitivity to dasatinib (a multikinase inhibitor) had a specific in vitro drug sensitivity pattern and gene expression signature, and were enriched for FLT3/ITD and PTPN11 mutations. Xenograft studies confirmed that dasatinib targets LSC in vivo.

\section{Methods}

\section{Primary acute myeloid leukemia cells}

Frozen mononuclear cells from bone marrow or peripheral blood of AML patients at diagnosis and relapse, when available, were used. Inclusion criteria for sample selection were achievement of complete remission. Patients with a low peripheral blood blast count and acute promeylocytic leukemia were excluded (Online Supplementary Table S1). Ethics approval: UHN 01-0573.

\section{Drug library}

We selected Food and Drug Administration-approved cancer drugs with demonstrated clinical and/or preclinical efficacy in leukemia and in other cancers. The 46 drugs and their mechanisms of action are detailed in Online Supplementary Table S2.

\section{Drug screening technology}

Cells were thawed, washed, counted and suspended in RPMI supplemented with $10 \%$ fetal calf serum and human cytokines: stem cell factor $(100 \mathrm{ng} / \mathrm{mL})$, interleukin $3(10 \mathrm{ng} / \mathrm{mL})$, interleukin $6(20 \mathrm{ng} / \mathrm{mL})$ and thrombopoietin $(10 \mathrm{ng} / \mathrm{mL})$. Sensitivity profiling was determined by dose-response over several log ranges (Online Supplementary Figure S1). Assay-ready plates in 384-well format were arrayed in 12 concentrations for each compound ( $2 \mathrm{nM}-50 \mu \mathrm{M})$, using a Labcyte Echo 555 acoustic dispenser, and frozen until use. Patients' samples were dispensed at approximately 1,000 cells per well and cultured for 2 days. Viable cells were quantified using Cell Titer Glo reagent (Promega Madison, WI, USA). Data were normalized using Genedata Screener software: dimethylsulfoxide-treated cells corresponded to $100 \%$ viability and samples without cells corresponded to $0 \%$ viability. Normalized data were loaded into CDD Vault software to calculate the half maximal inhibitory concentration $\left(\mathrm{IC}_{50}\right)$, and minimum and maximum responses for each drug and patient. We compared the in vitro response to dasatinib in our study with the results of the Beat AML drug sensitivity and resistance testing (DSRT). In the Beat AML study in vitro cultures were without cytokines. ${ }^{17}$

\section{RNA and exome sequencing}

The workflow for RNA sequencing was performed as previously described ${ }^{9}$ and the methods for RNA sequencing, exome sequencing, and gene expression and mutation analyses are detailed in the Online Supplementary Methods. For pathway analyses we used enrichr. ${ }^{19,20}$ 


\section{Prediction of response to dasatinib}

Dasatinib responders were defined in our cohort and in the Beat AML cohort as having an $\mathrm{IC}_{50}<0.01$, and dasatinib non-responders in both cohorts as having an $\mathrm{IC}_{50}>0.8$ (Online Supplementary Table S3). To identify predictors of response to dasatinib we initially selected genes that were differentially expressed (DE) between dasatinib responders and non-responders. Furthermore, other gene sets were selected based on pathway analysis of the $\mathrm{DE}$ genes and genes known to be targets of dasatinib as reported by the KinMap (Online Supplementary Table S4, genes with a $\mathrm{Kd}(\mathrm{nM})$ $<7$ [37 genes]). To refine feature selection, we tested which dasatinib target genes were DE between dasatinib responders and nonresponders from the Beat AML study (Online Supplementary Figure S3, Online Supplementary Table S4). Various machine-learning models were tested, all with a cross-validation of 10-fold, on the Beat AML data only. The best prediction model was the k-nearest neighbor algorithm with cosine similarity. This model was validated on the Israel National Center for Personalized Medicine (INCPM) gene expression data (Matlab statistical tool box).

\section{In vivo dasatinib treatment}

SGM3 mice were given an intrafemoral injection of $1 \times 10^{6} \mathrm{CD} 3-$ depleted AML human cells. From day 35 after the injection the mice were treated with $50 \mathrm{mg} / \mathrm{kg}$ dasatinib orally for 21 days and sacrificed on day 56. Fluorescence-activated cell sorting analysis was performed. Bone marrow cell suspensions were analyzed for expression of human CD45 (BV521, clone HL30), CD34 (APCcy7, clone 581), CD38 (PE cy7, clone 303516), CD15 (BV421, clone W6D3) (products from Biolegend), CD33 (APC), CD3 (FITC) and CD19 (PE) markers (products from Becton Dickinson). Human engraftment was defined as $>0.1 \%$ of cells positive for human CD45 at the time of sacrifice. The percent engraftment of human cells was compared between drug- versus control-treated mice using the Wilcoxon test. The Institutional Animal Care and Use Committee of the Weizmann Institute approved the experiments, which were performed in accordance with its relevant guidelines and regulations (11790319-2).

\section{Statistical analysis}

Heatmap and clustering of drug sensitivity analysis was performed with R package ComplexHeatmap. Differences between the defined groups were validated by statistical analysis. Enrichment analyses were performed using the Fisher exact test. Analysis of the DE genes was performed using the DESeq2 package $(1.10 .1)^{21}$ with the betaPrior, coks cutoff and independent filtering parameters set to "False". Raw $P$ values were adjusted for multiple testing using the procedure of Benjamini and Hochberg. DE genes were determined by an adjusted $P<0.05$, absolute fold changes $>2$ and maximum raw counts $>30$ (Figure 2, Online Supplementary Tables S5 and S6). Overlap between DE genes in the Beat AML and INCPM was tested by the hypergeometric distribution using the following $\mathrm{R}$ function sum (dhyper $[t: b, a, n-a$, b]). List a contains the DE genes in the Beat AML dataset, and $b$ the DE genes in INCPM. The total number of expressed genes in both datasets was 20,055. The intersection between $a$ and $b$ is $t$ (Figure 3A).

\section{Results}

\section{Classification of acute myeloid leukemia samples} based on drug response, and mutation profile

The overall goal of the current study was to identify drugs that can be safely given at diagnosis and target LSC. To achieve this goal, we first tried to identify drugs that were more effective at relapse than at diagnosis. Such drugs would presumably target LSC, which are enriched at relapse. Comparing the DSRT between paired AML diagnostic and relapse samples we found that for the majority of tested compounds the $\mathrm{IC}_{50}$ was higher for the relapse samples (Figure 1A), although the difference was statistically insignificant in most cases. Clinically relevant drugs that are being used to treat patients at relapse (etoposide, ${ }^{22}$ midostaurin ${ }^{23}$ and fludarabine ${ }^{24}$ ) were significantly less effective at relapse (Figure 1A). Next, we took an unbiased approach to identify subgroups of AML sensitive to specific drugs in order to identify patients likely to benefit from specific targeted therapies. We screened 29 primary AML samples by DSRT using a test set of 46 drugs. Five compounds (BEZ235, lenalidomide, visomodegib, EPZ-5676 and AG-120) were excluded from the analysis because of the low variability in the response among the different samples (almost all samples were resistant to these drugs). Unsupervised hierarchical clustering analysis of $\mathrm{IC}_{50}$ values demonstrated two distinct drug response patterns (Figure 1B). One group displayed high sensitivity to TKI (dasatinib, MEK inhibitors, PI3K inhibitor [BKM120], pim inhibitor, ruxolitinib, mTORC1/2 inhibitor [INK128], quizartinib, midostaurin), BET inhibitors (OTX015, JO1) and several other drugs. This group was termed sensitive. The other group was resistant to most compounds. The "resistant" group displayed heterogeneous sensitivity to BCL2 inhibitors (ABT737 and venetoclax), purine analogs (clofarabine, fludarabine,) and the hypomethylating agent decitabine. Interestingly, samples sensitive in vitro to BCL2 inhibitors (ABT-737 and venetoclax) did not cluster in a specific group, consistent with clinical observations (Figure 1B), 8,25 To define the molecular pathways underlying the drug sensitivity of the clustering groups, we analyzed the somatic mutations (exome sequencing) and gene expression of the AML samples. We focused our genetic analysis on genes that are recurrently mutated in AML. ${ }^{26}$ Mutations in FLT3/ATD were observed in seven of 17 patients in the "sensitive" group and none of 12 in the "resistant" group $(P=0.023)$. No other significant enrichment was identified in the current dataset.

\section{Gene expression and mutation profile \\ of dasatinib-sensitive acute myeloid leukemia cells}

Analysis of the in vitro DSRT assay identified a subgroup of AML samples sensitive to a broad range of TKI (Figure 1B). Among the TKI, the sensitivity to dasatinib was different between the two groups. In particular, 52\% (9 of 17) of the AML samples in the group of "responders" displayed sensitivity to dasatinib (defined as a 10-fold difference in $\mathrm{IC}_{50}$ between sensitive and non-sensitive samples) whereas in the group of "non-responders" none of the samples showed in vitro sensitivity to dasatinib $(P=0.003)$ (Figure 1B). Based on these results we focused our analysis on dasatinib, which has been safely used for many years as monotherapy or in combination with chemotherapy for Philadelphia-positive leukemia. Next, we tried to define those molecular features that could predict which AML samples would be sensitive to dasatinib. As our cohort was small, we compared our results to those of the Beat AML study. ${ }^{17}$ We classified dasatinib responders $(\mathrm{n}=61)$ and non-responders $(\mathrm{n}=134)$ based on $\mathrm{IC}_{50}$ values (Online Supplementary Table S3). Other parameters, including age, gender, leukocyte number, time of sample [diag- 
A

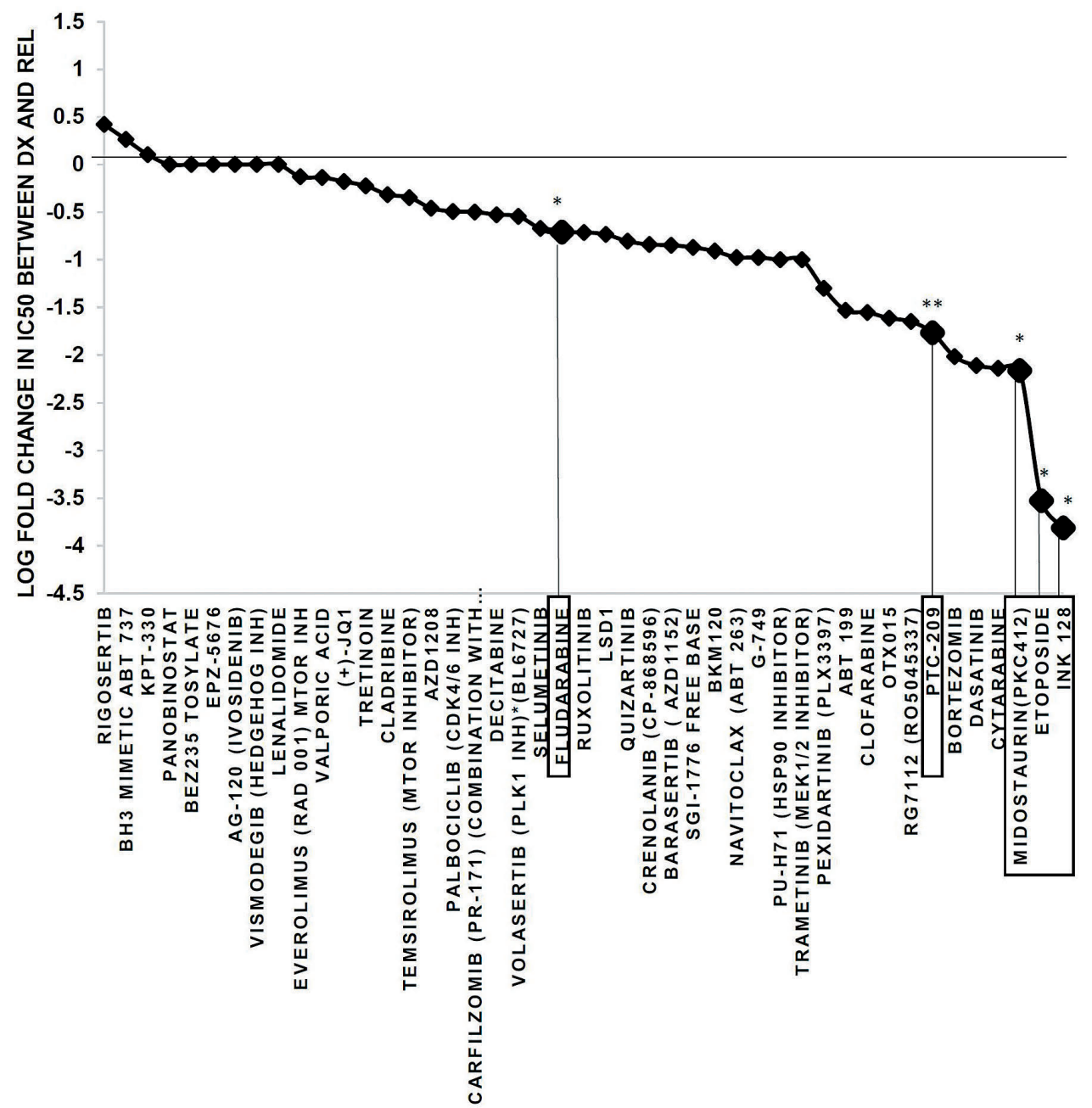

B

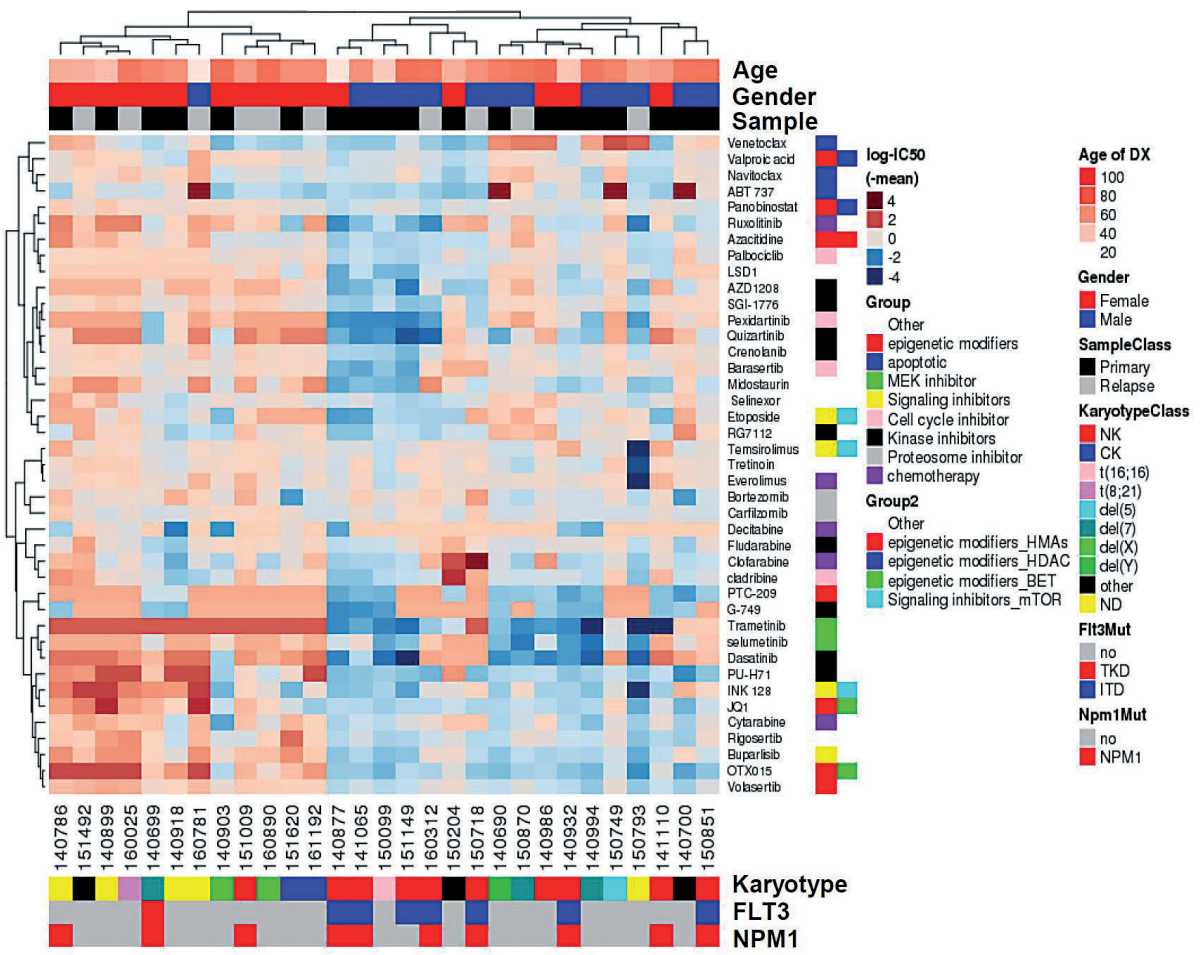

Figure 1. In vitro drug resistance. (A) In vitro drug resistance to most drugs is acquired after relapse. An average drug response of seven couples of primary acute myeloid leukemia (AML) samples, at diagnosis (DX) and relapse (REL) is shown, comparing the half maximal inhibitory concentration $\left(\mathrm{IC}_{50}\right)$ of 41 drugs. Each dot represents the response to a specific drug, calculated by the median $\mathrm{IC}_{50}$ ratio of diagnosis vs. relapse. (B) Drug sensitivity and resistance testing (DSRT) of primary AML cells. Hierarchical clustering using Pearson dissimilarity and complete linkage was performed. Data are the $\log _{2} I C_{50}$ +0.001 , standardized for each compound by reducing the mean. DSRT for 41 drugs of clinical and preclinical use in AML shows two clustered groups of patients. The age and gender of the patients and the origin of the patients' diagnostic or relapse sample, the name of the drugs, the class of the drug, karyotype and mutation status for FLT3/ITD and NPM1 are shown. 

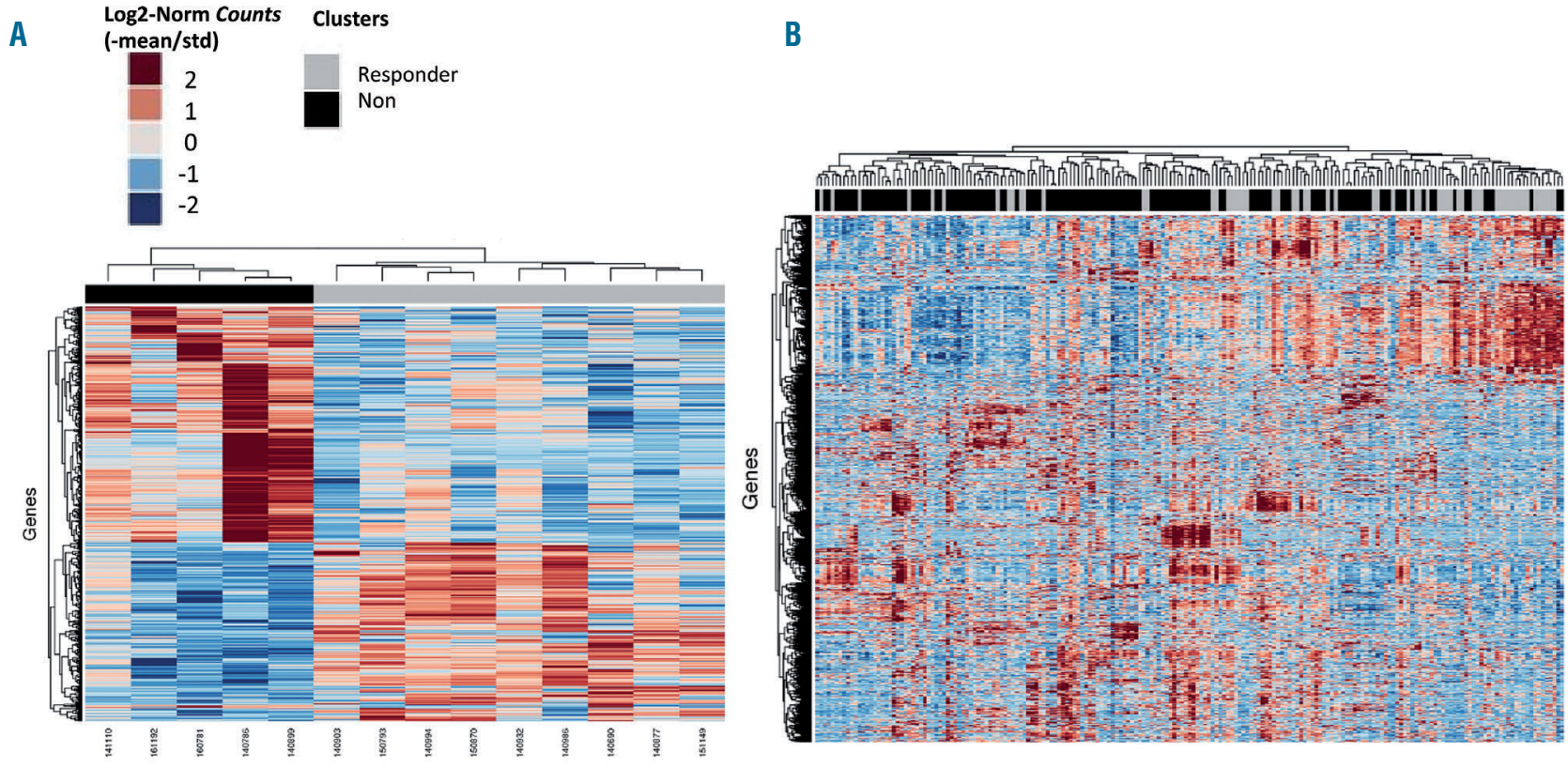

Figure 2. Transcriptome profiling of dasatinib responder samples. Gene expression analysis of whole transcriptome mRNA sequencing comparing dasatinib sensitive to non-sensitive samples of acute myeloid leukemia (AML). (A) Differentially expressed genes between "responders", and "non-responders". (B) In order to extend the tested samples we also applied the same analysis to AML patients' samples from the Beat AML dataset.

A

INCPM Up

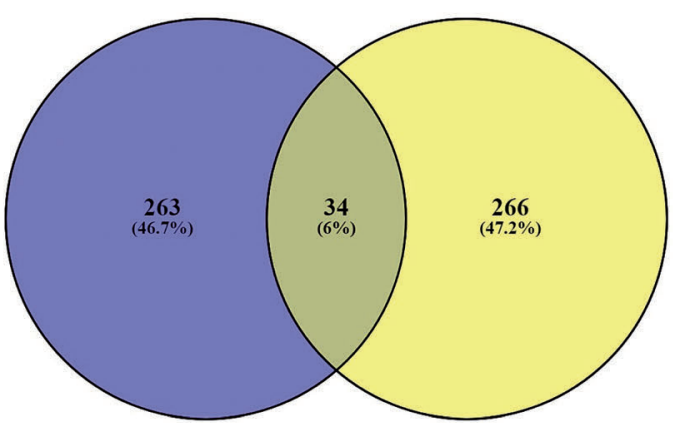

$P<\mathrm{e}-23$

INCPM Down

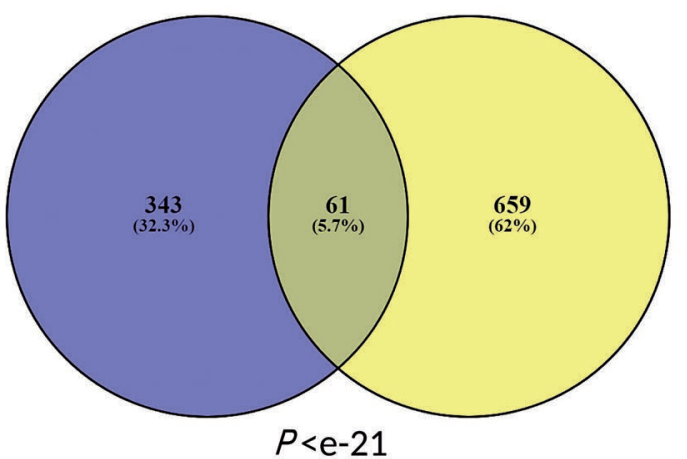

B

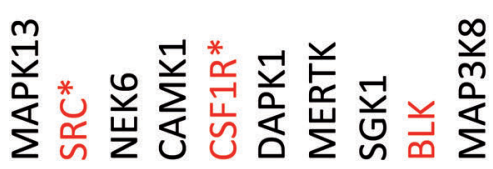

CD163

VSIG4

CYP27A1

C1QA

FOLR2

CPVL

HLA-DMB

ZNF385A

ARHGEF10L

PID1

KLF4

HLA-DRA

HLA-DPB1

SDS

NAPSB

FAM198B

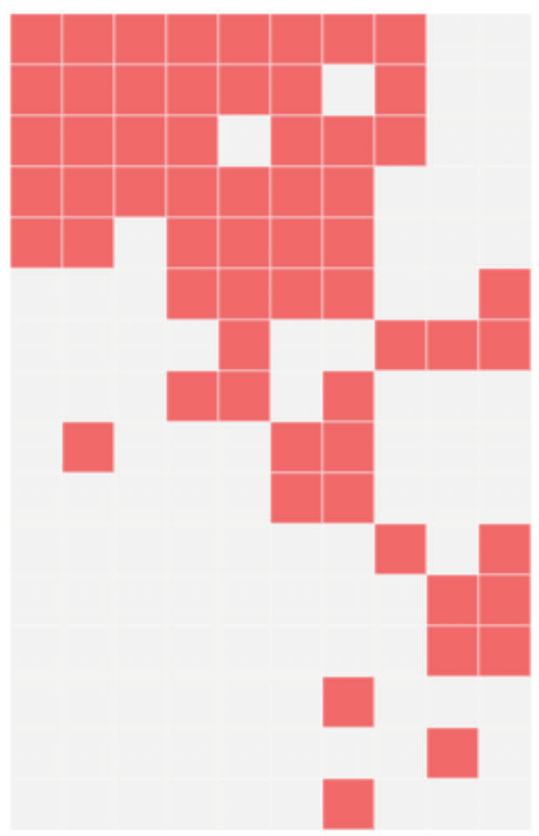

Figure 3. Analysis of differentially expressed genes between dasatinib responders and non-responders from the INCPM and Beat AML datasets. (A) Intersection of upregulated (upper panel) and downregulated (lower panel) differentially expressed genes from the INCPM and Beat AML cohorts. (B) Pathway analysis of intersecting upregulated genes in both cohorts identified significant enrichment of genes that are co-expressed with several dasatinib targets. Dasatinib targets (CSF1R, $S R C, B L K)$ are shown in red and the asterisk marks significant enrichment (false discovery rate<0.05). 
nosis/relapse], type of AML [de novo/transformed], karyotype and mutation profile were also tested for correlation with response to dasatinib.

Differential gene expression analysis of responders and non-responders to dasatinib in our cohort identified 297 genes significantly overexpressed and 404 genes downregulated in the sensitive group (false discovery rate $<0.05$ ) (Online Supplementary Table S5). In the Beat AML cohort, we identified 300 significantly upregulated and 720 downregulated genes in the dasatinib-sensitive group as compared to the resistant group (Online Supplementary Table S6). Unsupervised hierarchical clustering of all expressed genes enriched dasatinib responders in specific clusters both in our cohort (Figure 2A) and in the Beat AML cohort (Figure 2B). Intersecting the upregulated and downregulated DE genes from the Beat AML and the INCPM cohorts resulted in a significant number of intersecting genes
$\left(P<10^{-23}\right.$ and $P<10^{-21}$, respectively) (Figure $\left.3 \mathrm{~A}\right)$. Pathway analysis of intersecting upregulated genes in both cohorts identified significant enrichment of genes that are coexpressed with dasatinib targets (CSF1R, SRC) (Figure 3B, Online Supplementary Table S10). On the other hand, the same pathway analysis of intersecting downregulated genes did not enrich for dasatinib targets. As we identified upregulation of genes that are co-expressed with dasatinib targets we next performed a focused analysis on the expression of actual dasatinib targets. We identified all known dasatinib targets and performed unsupervised hierarchical clustering on their gene expression in both our cohort and the Beat AML cohort. In both cohorts a cluster enriched with dasatinib responders was identified. In the Beat AML cohort 13 of 21 samples (59\%) were responders in this cluster as opposed to 61 out of $195(31.3 \%)$ responders in the entire Beat AML cohort $(P=0.016)$ (Online
A

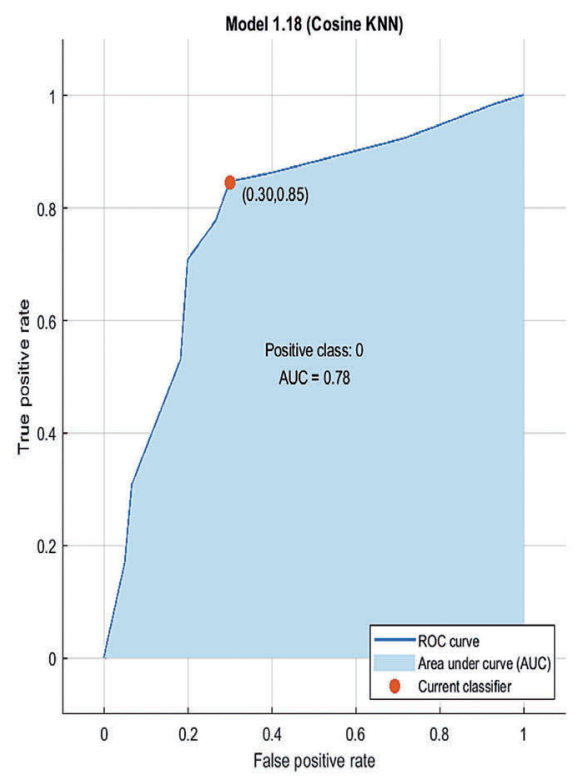

B

\section{Predicted Class}

Non-

Responders Responders

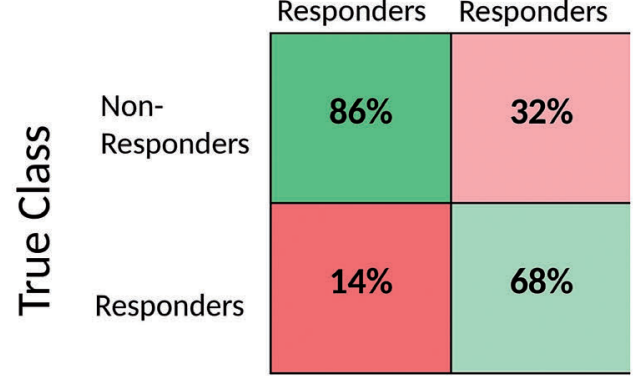

Positive Predictive Value

False Discovery Rate
Figure 4. Prediction model to identify dasatinib responders based on gene expression. (A, B) The k-nearest neighbor (KNN) algorithm with cosine similarity was used to predict the response to dasatinib. The greatest accuracy was achieved with 10fold cross-validation applied to the differentially downregulated genes from the Beat AML dataset. Accordingly, a sensitivity of 0.85 and a specificity of 0.7 were achieved. (C) Validation of the KNN cosine prediction model on INCPM data.
C

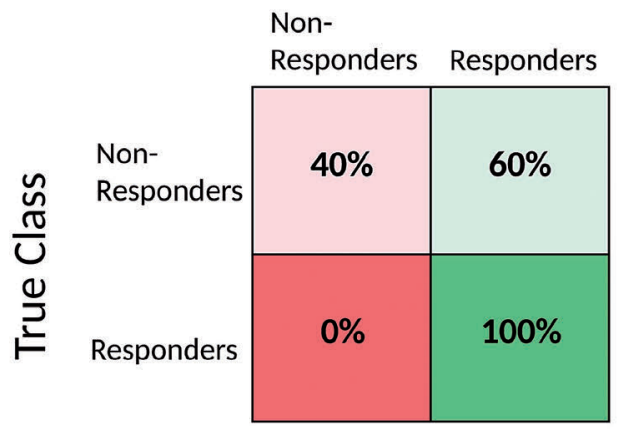

Positive Predictive Value

False Discovery Rate

\begin{tabular}{|c|c|}
\hline $40 \%$ & $100 \%$ \\
\hline $0 \%$ & $60 \%$ \\
\hline
\end{tabular}


Supplementary Figure S2). In both cohorts the dasatinib target cluster included three SRC family tyrosine kinases FGR, HCK and LYN as well as PTK6, CSK, GAK and $E P H B 2$. Several of these genes were significantly overexpressed in dasatinib responders in the Beat AML cohort (Online Supplementary Figure S3).

Next, we used the data from the gene expression analysis to establish a prediction model for identifying dasatinib responders in vitro based on gene expression. For all experiments we used a cross-validation approach, training the model only on the Beat AML data and validating results on the INCPM data. We tested the following genes sets from the Beat AML data for prediction: all genes, all DE genes, all upregulated genes, all downregulated genes, all dasatinib targets and DE dasatinib targets. The best prediction model, with a sensitivity of 0.85 and specificity of 0.7 (area under the curve $=0.78$ ) (Figure 4A,B), was achieved when using the Beat AML downregulated genes. When testing the same model on the INCPM data the positive predictive value was $100 \%$ and the false discovery rate was $60 \%$ (Figure $4 \mathrm{C}$ ). These data provide evidence that gene expression can predict in vitro response to dasatinib, albeit with limited accuracy.

As gene expression did not provide an ideal prediction for dasatinib response, we tested whether any other parameters from the Beat AML could predict response to dasatinib. We analyzed molecular and clinical data for enrichment in dasatinib responders. We found that samples carrying mutations in FLT3/ITD $(P=0.011)$ (Online Supplementary Table S7), IDH2 and PTPN11 $(P<0.05)$ were enriched in dasatinib responders in the Beat AML cohort. The $\mathrm{IC}_{50}$ for dasatinib in carriers of FLT3/ITD and PTPN11 was significantly lower than the $\mathrm{IC}_{50}$ of non-carriers $(P<0.0001$ and $P=0.02$, respectively) (Figure 5A, B and Online Supplementary Table S8), whereas only samples with mutations in TP53 had significantly higher $\mathrm{IC}_{50} \mathrm{com}-$ pared to wild-type samples $(P=0.0003)$ (Figure 5C and Online Supplementary Table S9). Adding the mutation status to the gene expression prediction model did not improve accuracy.

Collectively, these data suggest that molecular features (mutations and gene expression profile) identify a group of AML cases responsive to dasatinib in vitro. Specifically, the fact the FLT3/ITD mutant cases are enriched in dasatinib responders in both the Beat AML and INCPM cohorts prompted us to test the in vivo efficacy of dasatinib in this specific group of patients. As our ultimate goal was to test whether in vitro response to dasatinib would predict in vivo AML response, we chose to focus our in vivo dasatinib response studies on samples known to carry FLT3/ATD. The focus on FLT3/ITD also stemmed from the fact that FLT3/ITD AML samples are known to be good AML engrafters. $^{27}$

Dasatinib reduces leukemia stem cells in mice engrafted with FLT3/ITD-mutant acute myeloid leukemia

We injected NSG mice ( $\mathrm{n}=5-10 /$ sample) with cells from nine patients with FLT3/ITD AML. On day 35 the animals were randomized to dasatinib or a carrier control. Following 3 weeks of treatment we measured human engraftment in the treated and control groups (Figure 6). Fluorescence-activated cell sorting of bone marrow demonstrated leukemic engraftment $\left(\mathrm{CD}_{3} 3^{+}>90 \%\right.$ of human $\mathrm{CD}^{4} 5^{+}$cells) in mice from both groups. For four of nine samples, engraftment was significantly reduced in the dasatinib-treated group. This result suggests that dasatinib has anti-LSC activity in a subset of FLT3/ITD positive AML cases.

A

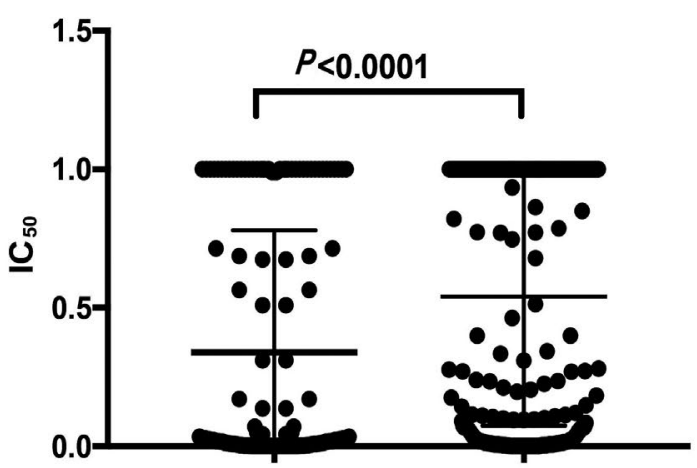

Mutated WT

FLT3-ITD

B

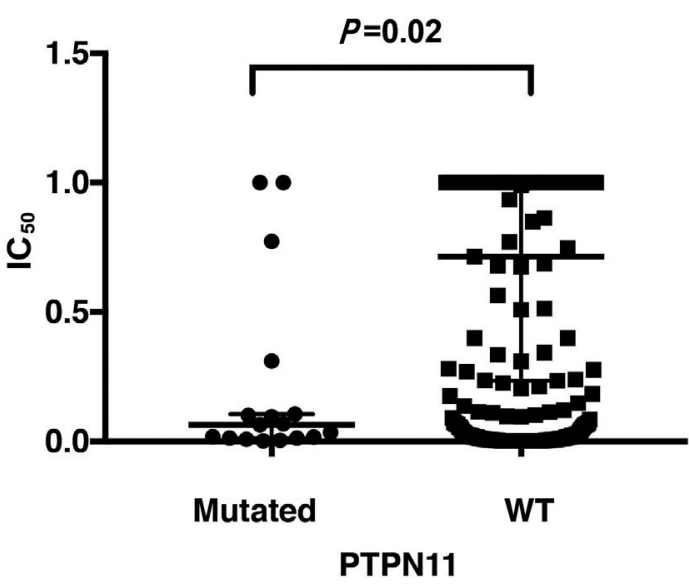

C

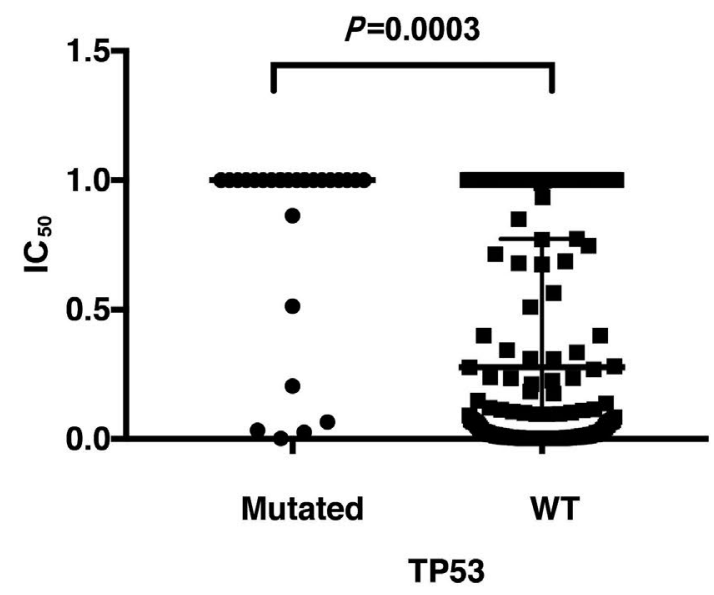

Figure 5. Correlation between FLT3/ITD, PTPN11 and TP53 mutations in acute myeloid leukemia samples and response to dasatinib in vitro in the Beat AML study. (A-C) Median dasatinib half maximal inhibitory concentration $\left(\mathrm{IC}_{50}\right)$ values in cases with mutated FLT3/ITD (A), PTPN11 (B) or TP53 (C) and wild-type (WT) samples. Differences between groups were validated by the Wilcoxon rank-sum test. 


\section{Discussion}

We provide evidence that a subset of FLT3/ITD ${ }^{+}$AML cases and AML subtypes with a unique gene expression pattern are sensitive to dasatinib in vitro and that this sensitivity translates into sensitivity in vivo, at least in FLT3/ITD ${ }^{+}$cases. We initially discovered sensitivity to dasatinib using DSRT and validated the DSRT results based on the Beat AML study. DSRT can only measure toxicity to bulk blast cells and cannot detect effects on differentiation and LSC. Accordingly, we tested the response of FLT3/ITD ${ }^{+}$primary AML samples to dasatinib in a xenograft model and confirmed response in vivo.

FLT3/ITD is one of the most common mutations in AML, and is associated with high risks of relapse and mor-
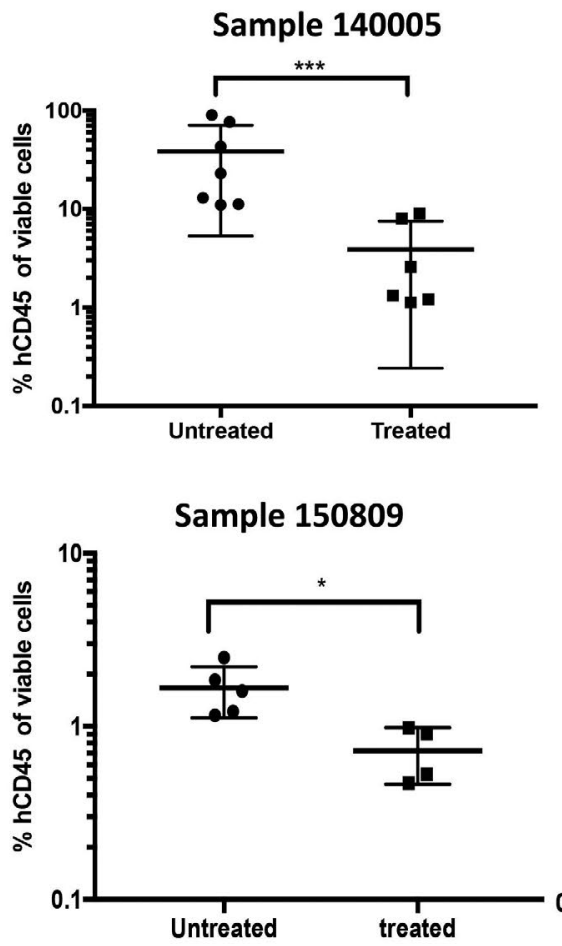

Sample 150279

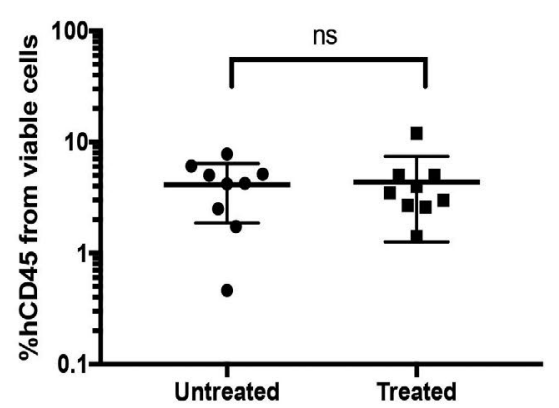

Sample 160436

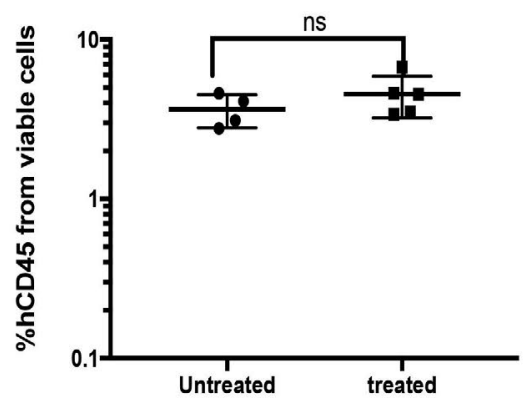

Sample 40094

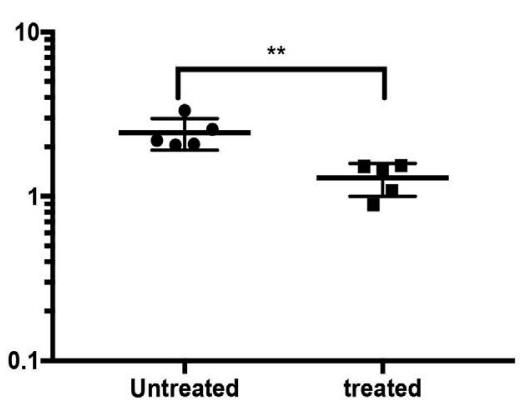

Sample 40034

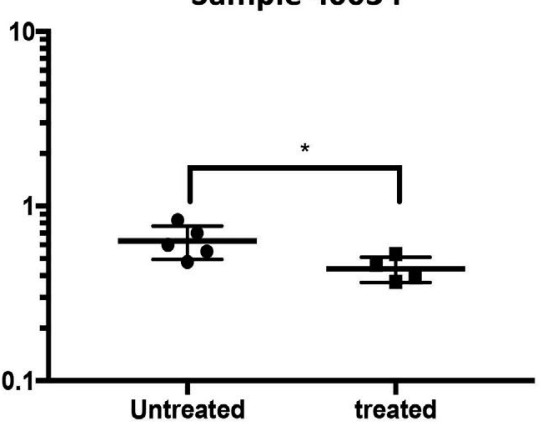

Sample 130695

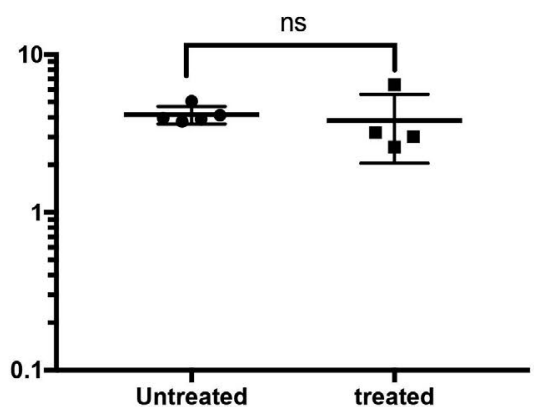

Sample 160406

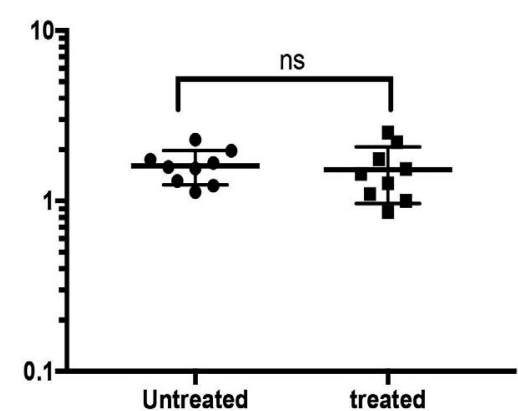

Figure 6. Dasatinib delays development of human FLT3/ITD-mutated acute myeloid leukemia in transplanted mice. SGM3 mice were treated with dasatinib for 3 weeks after transplantation of human cells from nine patients with FLT3/ITD acute myeloid leukemia (AML). The percentage of human $\mathrm{CD}^{4} 5^{+}$(hCD45) cells in engrafted murine bone marrow with/without dasatinib treatment is shown after staining for CD45, CD34, CD33, CD38, CD15, CD3 and CD19 to determine myeloid lineage cells and analyzed by fluores cence-activated cell sorting immunostaining. Wilcoxon rank sum test, $* P<0.05$; $* * P<0.005 ; * * * P<0.0005$. The mutation status of the AML samples was: 140005: FLT3/ITD ${ }^{+}$FLT3/TKD, NPM1+; 40094: NPM1+, FLT3/ITD ${ }^{+}$150809: NPM1; FLT3/ITD'; 40034: NPM1, FLT3/ITD ${ }^{+}, 150279$ : FLT3/ITD ${ }^{+}$, FLT3/TKD, NPM1+; 130695 NPM1 ${ }^{+}, \quad F L T 3 /$ ITD $^{+}, \quad F L T 3 / T K D ; 160436:$ NPM1+, FLT3/ITD ${ }^{+}$; 160406: FLT3/ITD ${ }^{+}$, NPM1 ${ }^{+} ; 130607:$ FLT3/ITD ${ }^{+}$, NPM $^{+}$.
Sample 130607

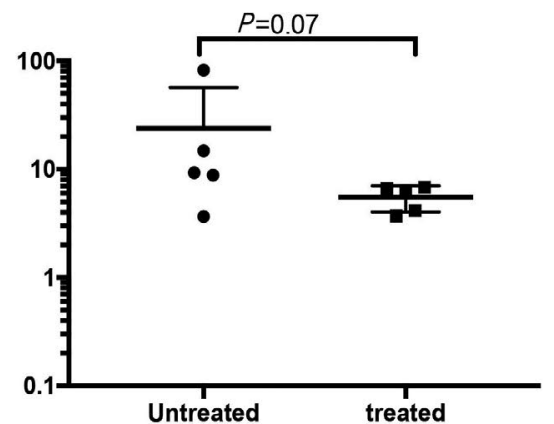


tality. ${ }^{28-31}$ Recently the introduction of FLT3 TKI has improved overall survival; ;2,33 however, not all FLT3/ITD ${ }^{+}$ patients respond to FLT3 inhibitors, whereas some FLT3/ITD patients do. ${ }^{34}$ We show that FLT3/ITD AML cells are sensitive to dasatinib both in vitro and in xenograft models. FLT3/ITD results in constitutive activation of FLT3 and activates several signal transduction pathways such as RAS-MAPK, PIBK, JAK-STAT and SRC. This broad signal transduction activation might explain the heterogeneity observed in the response to FLT3 inhibitors. The current standard of care for patients with FLT3/ITD is midostaurin combined with chemotherapy. However, in the RATIFY study only $46.4 \%$ of patients achieving complete remission after midostaurin remained disease-free after 4 years with an ongoing risk of relapse, especially during the first year after treatment. ${ }^{23}$ The high failure rate even following combination therapy indicates that additional therapy is needed upfront. Previous studies by Weisberg et al. demonstrated synergistic effects for the combination of midostaurin and dasatinib in FLT3/ITD mutated AML cell lines. ${ }^{35}$ Our in vivo data demonstrate that the anti-leukemic activity of dasatinib extends to FLT3/ITD LSC, at least for a subset of patients, and suggest that addition of dasatinib to the current standard of care may be beneficial for FLT3/ITD AML patients.

Our results also show that a unique gene expression signature correlates with response to dasatinib. Consistent with mechanistic predictions dasatinib responders overexpressed multiple dasatinib targets. SRC family kinase (SFK) genes, such as $L Y N, H C K^{36}$ and FGR (which are all dasatinib targets) are overexpressed in dasatinib responders in the Beat AML dataset (Figure 3B, Online Supplementary Figure S3), and were shown to be overexpressed in AML-LSC and to contribute to the survival and proliferation of these cells. ${ }^{37}$ Exposure to dasatinib treatment inhibited SFK phosphorylation in primitive and committed AML progenitors. In this study the combination of dasatinib and chemotherapy enhanced LSC targeting by p53 signaling. ${ }^{37}$ Furthermore recent evidence suggests that FLT3 activates SFK and these kinases in turn regulate the activity of the RAS/ERK pathways. ${ }^{38}$ Altogether, our data suggest that dasatinib may be active not only in FLT3/ITD AML but also in other subtypes of AML sharing a similar dasatinib responsive gene expression signature. Similarly, we showed that dasatinib may be effective in treating PTPN11-mutated AML, but likely not all PTPN11-mutated AML will respond to dasatinib and a larger cohort is needed to identify additional biomarkers. Mutations in PTPN11 are also found in 35\% of patients with juvenile myelomonocytic leukemia and acute lymphocytic leukemia, and in $4-7 \%$ of $\mathrm{AML}$ patients. PTPN11 encodes the Shp2 cytoplasmatic protein tyrosine phosphatase, and SHP2 mutations cause increased phosphatase activity that contributes to leukemogenesis by upregulating the RAS, JAK-STAT, and PI3K pathways, leading to aberrant proliferation of myeloid progenitors. ${ }^{39,40}$ A recent study demonstrated that TNK2 activates PTPN11; furthermore, mutant myeloid leukemic cells carrying PTPN11-activating mutations were sensitive to TNK inhibition by dasatinib in vitro. ${ }^{41}$ Future studies are needed to better define the subset of PTPN11-mutated AML likely to respond to dasatinib and to test the activity of dasatinib activity in vivo.

Our results suggest that the addition of dasatinib to the current standard of care for FLT3/ITD AML (an FLT3 inhibitor in combination with cytarabine/anthracycline) may benefit a subset of FLT3/ITD ${ }^{+}$patients and should be tested in a clinical trial. Similarly, patients ineligible for intensive induction therapy may benefit from combining dasatinib, FLT3 TKI, hypomethylating agents and venetoclax. Clearly the potential toxicity of such combinations needs to be taken into account. Another possible clinical trial would be to investigate the addition of dasatinib to standard of care in patients with PTPN11-mutated AML. A unique gene expression signature, as detected by RNAsequencing, may predict response to dasatinib, suggesting that selection of patients based on RNA-sequencing may be included in future clinical trials. Given dasatinib's activity against LSC, there is hope that addition of dasatinib during initial therapy will reduce the risk of subsequent relapse.

\section{Acknowledgments}

This research was supported by a grant from the Nancy and Stephen Grand Israel National Center for Personalized Medicine to $H B$ and by the EU horizon 2020 grant project MAMLE ID: 714731, LLS grant ID: RTF6005-19, and IMOS-712843 awarded to LIS. LIS is an incumbent of the Ruth and Louis Leland career development chair. NK is an incumbent of the Applebaum Foundation Research Fellow Chair. This research was also supported by the Sagol Institute for Longevity Research, the Barry and Eleanore Reznik Family Cancer Research Fund, Steven B. Rubenstein Research Fund for Leukemia and Other Blood Disorders, the Rising Tide Foundation and the Applebaum Foundation.

\section{References}

1. Tuval A, Shlush LI. Evolutionary trajectory of leukemic clones and its clinical implications. Haematologica. 2019;104(5):872-880.

2. McMahon CM, Perl AE. Gilteritinib for the treatment of relapsed and/or refractory FLT3mutated acute myeloid leukemia. Expert Rev Clin Pharmacol. 2019;12(9):841-849.

3. Perl AE. The role of targeted therapy in the management of patients with AML. Blood Adv. 2017;1(24):2281-2294.

4. Stone RM, Larson RA, Dohner H. Midostaurin in FLT3-mutated acute myeloid leukemia. N Engl J Med. 2017;377(19):1903.

5. DiNardo CD. Ivosidenib in IDH1-mutated acute myeloid leukemia. N Engl J Med. 2018;379(12):1186
6. Stein EM, DiNardo CD, Pollyea DA, et al Enasidenib in mutant IDH2 relapsed or refractory acute myeloid leukemia. Blood. 2017;130(6):722-731

7. Bisaillon R, Moison C, Thiollier C, et al. Genetic characterization of ABT-199 sensitivity in human AML. Leukemia. 2020;34(1): 63-74.

8. Pollyea DA, Stevens BM, Jones CL, et al. Venetoclax with azacitidine disrupts energy metabolism and targets leukemia stem cells in patients with acute myeloid leukemia. Nat Med. 2018;24(12):1859-1866

9. Shlush LI, Mitchell A, Heisler L, et al. Tracing the origins of relapse in acute myeloid leukaemia to stem cells. Nature. 2017;547(7661):104-108.

10. Shlush LI, Chapal-Ilani N, Adar R, et al. Cell lineage analysis of acute leukemia relapse uncovers the role of replication-rate heterogeneity and microsatellite instability. Blood. 2012;120(3):603-612.

11. Ding L, Ley TJ, Larson DE, et al. Clonal evolution in relapsed acute myeloid leukaemia revealed by whole-genome sequencing. Nature. 2012;481(7382):506-510.

12. Estey E, Levine RL, Lowenberg B. Current challenges in clinical development of "targeted therapies": the case of acute myeloid leukemia. Blood. 2015;125(16):2461-2466

13. Eriksson A, Osterroos A, Hassan S, et al Drug screen in patient cells suggests quinacrine to be repositioned for treatment of acute myeloid leukemia. Blood Cancer J. 2015;5:e307.

14. Snijder B, Vladimer GI, Krall N, et al. Imagebased ex-vivo drug screening for patients with aggressive haematological malignan- 
S. Favor et al.

cries: interim results from a single-arm, openlabel, pilot study. Lancet Haematol. 2017;4(12):e595-e606.

15. Pemovska T, Kontro M, Yadav B, et al. Individualized systems medicine strategy to tailor treatments for patients with chemorefactory acute myeloid leukemia. Cancer Discov. 2013;3(12):1416-1429.

16. Bose P, Vachhani P, Cortes JE. Treatment of Relapsed/Refractory Acute Myeloid Leukemia. Cor Treat Options Oncol. 2017;18(3):17.

17. Tyner JW, Tognon CE, Bottomly D, et al. Functional genomic landscape of acute myeloid leukaemia. Nature. 2018;562(7728): 526-531.

18. Gerstung M, Papaemmanuil E, Martincorena I, et al. Precision oncology for acute myeloid leukemia using a knowledge bank approach. Nat Genet. 2017;49(3):332-340.

19. Chen EY, Tan CM, Kou Y, et al. Enrich: interactive and collaborative HTML5 gene list enrichment analysis tool. BMC Bioinformatics. 2013;14:128.

20. Kuleshov MV, Jones MR, Rouillard AD, et al. Enrich: a comprehensive gene set enrichment analysis web server 2016 update. Nucleic Acids Res. 2016;44(W1): W90-97.

21. Anders S, McCarthy DJ, Chen Y, et al. Count-based differential expression analysis of RNA sequencing data using $R$ and Bioconductor. Nat Protoc. 2013;8(9):17651786.

22. Megias-Vericat JE, Martinez-Cuadron D, San MA, et al. Salvage regimens using conventional chemotherapy agents for relapsed/refractory adult AML patients: a systematic literature review. Ann Hematol. 2018;97(7):1115-1153.

23. Stone RM, Mandrekar SJ, Sanford BL, et al. Midostaurin plus chemotherapy for acute myeloid leukemia with a FLT3 mutation. N Engl J Med. 2017;377(5):454-464.

24. Fiegl M, Unterhalt M, Kern W, et al. Chemomodulation of sequential high-dose cytarabine by fludarabine in relapsed or refractory acute myeloid leukemia: a ran- domized trial of the AMLCG. Leukemia. 2014;28(5):1001-1007.

25. DiNardo CD, Pratz K, Pullarkat V, et al. Venetoclax combined with decitabine or azacitidine in treatment-naive, elderly patients with acute myeloid leukemia. Blood. 2019;133(1):7-17.

26. Papaemmanuil E, Gerstung M, Bullinger L, et al. Genomic classification and prognosis in acute myeloid leukemia. N Engl J Med. 2016;374(23):2209-2221.

27. Sanchez PV, Perry RL, Sorry JE, et al. A robust xenotransplantation model for acute myeloid leukemia. Leukemia. 2009;23(11): 2109-2117.

28. Kottaridis PD, Gale RE, Frow ME, et al. The presence of a FLT3 internal tandem duplicaion in patients with acute myeloid leukemia (AML) adds important prognostic information to cytogenetic risk group and response to the first cycle of chemotherapy: analysis of 854 patients from the United Kingdom Medical Research Council AML 10 and 12 trials. Blood. 2001;98(6):17521759.

29. Yanada M, Matsuo K, Suzuki T, et al. Prognostic significance of FLT3 internal tandem duplication and tyrosine kinase domain mutations for acute myeloid leukemia: a meta-analysis. Leukemia. 2005;19(8):13451349.

30. Thiede C, Strudel C, Moho B, et al. Analysis of FLT3-activating mutations in 979 patients with acute myelogenous leukemia: associatimon with FAB subtypes and identification of subgroups with poor prognosis. Blood. 2002;99(12):4326-4335.

31. Kayser S, Schlenk RF, Londono MC, et al. Insertion of FLT3 internal tandem duplicaton in the tyrosine kinase domaine is lassocrated with resistance to chemotherapy and inferior outcome. Blood. 2009;114(12):23862392.

32. Sasaki K, Kantarjian HM, Kadia T, et al. Sorafenib plus intensive chemotherapy improves survival in patients with newly diagnosed, FLT3-internal tandem duplicaion mutation-positive acute myeloid leukemia. Cancer. 2019;125(21):3755-3766.

33. Uy GL, Mandrekar SJ, Lawman K, et al. A phase 2 study incorporating sorafenib into the chemotherapy for older adults with FLT3-mutated acute myeloid leukemia: CALGB 11001. Blood Adv. 2017;1(5):331340.

34. Rolling C, Serve H, Huttmann A, et al. Addition of sorafenib versus placebo to stanlard therapy in patients aged 60 years or younger with newly diagnosed acute myeloid leukaemia (SORAML): a multicenthe, phase 2, randomised controlled trial. Lancet Oncol. 2015;16(16):1691-1699.

35. Weinberg E, Lu Q, Nelson E, et al. Using combination therapy to override stromalmediated chemoresistance in mutant FLT3positive AML: synergism between FLT3 inhibitors, dasatinib/multi-targeted inhibitors and JAK inhibitors. Leukemia. 2012;26(10):2233-2244.

36. Salto Y, Kitamura H, Hijikata A, et al. Identification of therapeutic targets for guiscent, chemotherapy-resistant human leukemia stem cells. Sci Transl Med. 2010;2(17):17ra9.

37. Dos Santos C, McDonald T, Ho YW, et al. The Sic and c-Kit kinase inhibitor dasatinib enhances p53-mediated targeting of human acute myeloid leukemia stem cells by chemotherapeutic agents. Blood. 2013;122 (11):1900-1913.

38. Kazi JU, Ronnstrand L. The role of SRC famfly kinase in FLT3 signaling. Int J Biochem Cell Biol. 2019;107:32-37.

39. Li L, Modi H, McDonald T, et al. A critical role for SHP2 in STAT5 activation and growth factor-mediated proliferation, survival, and differentiation of human CD 34+ cells. Blood. 2011;118(6):1504-1515.

40. Schubert S, Lieut K, Rowe SL, et al. Functional analysis of leukemia-associated PTPN11 mutations in primary hematopoietic cells. Blood. 2005;106(1):311-317.

41. Jenkins C, Luty SB, Mason JE, et al. Synthetic lethality of TNK2 inhibition in PTPN11-mutant leukemia. Sci Signal. 2018;11(539):eaao5617.

2804

haematological | 2020; 105(12) 